\title{
LOS COMIENZOS OFICIALES DEL USO DE LAS NUEVAS TECNOLOGÍAS DE LA INFORMACIÓN Y LA COMUNICACIÓN EN LA EDUCACIÓN COLOMBIANA ${ }^{1}$
}

\author{
Jorge E. Benavides B. ${ }^{2}$ \\ Universidad de Nariño - Colombia \\ Grupo de Investigación HISULA \\ jbenavides@udenar.edu.co
}

Recepción: 04/09/2012

Evaluación: 29/09/2012

Aceptación: 15/11/2012

Artículo de Reflexión

doi: http://dx.doi.org/10.9757/Rhela.19.01

\section{RESUMEN}

Este estudio intenta una aproximación a la historia reciente de la introducción discursiva de las Nuevas Tecnologías de la Información y la Comunicación (NTIC) por parte del gobierno colombiano de inicio de los años ochenta. Su objetivo fue determinar dentro de los mecanismos oficiales de implementación de la tecnología informática los recursos documentales y discursivos para la política de modernización del Estado como parte de la preocupación por el desarrollo social, económico y educativo en el contexto de un mundo de tendencia neoliberal globalizante. Se investigaron las principales instancias documentales que expresaban las motivaciones e intereses que dieron origen formal a la introducción principalmente teórica y discursiva de los desarrollos tecnológicos del mundo en proceso de globalización neoliberal en el sistema estatal colombiano. Se encontró que la política oficial de estos comienzos estuvo determinada por la presión para la modernización del aparato estatal y la urgencia por la integración al mundo interconectado de inicios globalizantes, a través de la creación de diferentes entidades e instituciones administrativas oficiales encargadas de la introducción de las nuevas tecnologías de la información para la capacitación del recurso humano, especialmente en educación.

Palabras clave: Revista Historia de la Educación Latinoamericana, NTIC, nuevas tecnologías de la información y la comunicación, políticas oficiales, educación, modernización, globalización.

\footnotetext{
1 Este estudio se realizó, principalmente en su base teórica, con el apoyo de la Universidad de Narińo para la pasantía que el autor como investigador invitado realizó en Central Washington University, Ellensburg, U.S.A. (Octubre-Diciembre, 2005) durante el programa de Doctorado.

2 Docente titular e investigador adscrito al Departamento de Lingüística e Idiomas de la Universidad de Nariño, San Juan de Pasto, Colombia. Doctor en Ciencias de la Educación. Universidad de Nariño-RUDECOLOMBIA, 2010. Director del grupo de investigación TICED reconocido por COLCIENCIAS (convocatoria, 2012).
} 


\section{USE OF INFORMATION AND COMMUNICATION TECHNOLLOGIES AND ITS OFICIAL BEGINING IN COLOMBIAN EDUCATION}

\section{ABSTRACT}

This paper aims an approximation towards history of new Technologies of Information and Communication (NICT) inclusion into Colombia's Goverment speech since the beginning of 80's. Their objective was to determinate a way to implement information technology in the official channels for both archives and speech for state's modernization policy this was concerning to social, economic and academical development during a neo liberal context that tended towards globalization.

Research was based on main document sources that expressed motives and interests that originated a mainly theorical and speech introduction of technological development following a neo liberal state that was heading towards globalization.

There was a remarkable result on this decade's official policy that was determinated by a special presure for modernising Se encontró que la política oficial de estos comienzos estuvo determinada por la presión para la modernización del aparato estatal y la urgencia por la integración al mundo interconectado de inicios globalizantes, a través de la creación de diferentes entidades e instituciones administrativas oficiales encargadas de la introducción de las nuevas tecnologías de la información para la capacitación del recurso humano, especialmente en educación.

Key words: History of Latin American Education Journal, NICT, new information technologies and communication, government policies, education, modernization, globalization.

\section{AS ORIGENS OFICIAIS DO USO DAS NOVAS TECNOLOGIAS DA INFORMAÇÃO NA EDUCAÇÃO COLOMBIANA}

\section{RESUMO}

Este estudo tem o objetivo de analisar a história recente da introdução discursiva das Novas Tecnologias da Informação e Comunicação (NTIC) por parte do governo colombiano desde o início da década de 1980 . O objetivo foi determinar, a partir dos mecanismos oficiais de implementação da tecnologia informática, dos recursos documentais e discursivos para a política de modernização do estado como parte da preocupação pelo desenvolvimento nacional, econômico e educativo no contexto de um mundo de tendência neoliberal e globalizante. Foram pesquisadas as principais instâncias documentais que expressavam as motivações e os interesses que deram origem formal à introdução, principalmente teórica e discursiva, dos desenvolvimentos tecnológicos do mundo em processo de globalização neoliberal no sistema estatal colombiano. Foi possível concluir que a política oficial destas origens esteve determinada pela pressão para a modernização do aparato estatal e urgência pela integração ao mundo interconectado de princípios globalizantes, através da criação de diferentes entidades e instituições administrativas oficiais encarregadas da introdução das novas tecnologias da informação para a capacitação do recurso humano, especialmente em educação.

Palavras-chave: Revista História da Educacão Latino-americana, NTIC, novas tecnologias da informação e da comunicação, políticas oficiais, educação, modernização, globalização.

\section{INTRODUCCIÓN}

En fecha no tan lejana como el siglo XVIII, el enciclopedismo hacía notar que llegaría una época en que aprender algo de los libros sería casi tan normal como estudiar directamente del universo entero; que la proliferación de la imprenta y su papel extensivo llenaría de libros las bibliotecas para los lectores ávidos de información y de conocimiento y que finalmente, el mundo del aprendizaje sería un mundo de libros. 
Sin embargo, en nuestro mundo más reciente ya no son solo los libros en las bibliotecas los únicos medios disponibles para acceder a la información y al conocimiento sino todos los medios informáticos que ha impuesto la tecnología informática moderna a través de los cuales se puede tener acceso a la información para una multiplicidad de propósitos. Este proceso de introducción, apropiación e integración de las nuevas tecnologías de la información y la comunicación (NTIC, en adelante) -especialmente en lo que se refiere a su capacidad de proporcionar a los individuos de acceso nuevo e ilimitado al universo de la información- ha sido tan dramático que todavía no se sabe del todo cuándo se inició y cómo se ha producido.

A nivel internacional se ha llegado a considerar que las características de las nuevas tecnologías son compatibles con las de los centros especializados de estudio y aprendizaje de las universidades más afamadas del mundo y que algunas de las capacidades más significativas de estas se parecen a las capacidades de las bibliotecas de las universidades dedicadas especialmente a la investigación y que encajan funcionalmente con ellas. Así como las bibliotecas han sido para la investigación un instrumento de aprendizaje y de conocimiento extremadamente poderoso, y útil para el desarrollo de la educación y de la cultura, también lo han estado siendo durante estas dos últimas décadas las NTIC e Internet -y en gran parte por las mismas razones.

Para comprender mejor el desarrollo de estos procesos de la introducción e integración de las NTIC en el desarrollo de los países, y particularmente en el sistema educativo colombiano, es necesario preguntarse sobre los orígenes oficiales y documentales de la introducción de estas nuevas tecnologías en la historia reciente del país a nivel general y en particular las gestiones administrativas iniciales de su implementación dentro del contexto histórico en el que se llevaron a cabo.

\section{La historia reciente de las NTIC en los documentos oficiales}

Es importante considerar dentro del aspecto metodológico de la historia las observaciones que se han hecho sobre los documentos oficiales como fuente de investigación histórica. En este sentido dentro de los 
métodos y técnicas de investigación histórica, Julio Aróstegui integra al campo de la investigación cualitativa la observación documental y dentro de esta, el análisis de la producción de documentos oficiales como fuentes primarias. Considera también al documento y su discurso como símbolo, entre las fuentes primarias o secundarias, sobre las cuales se construye la historia, en tanto "... discurso intelectual que procede de la creatividad humana, cuyo través puede inferirse algo acerca de una determinada situación social en el tiempo"3.

Por consiguiente el enfoque de aproximación a los documentos y su análisis constituye un paradigma cualitativo e interpretativo de investigación puesto que el objeto se relaciona con la observación y descripción de un fenómeno de cambio e innovación educativa a través del análisis documental donde la naturaleza de los acontecimientos discursivos implica la necesidad de un 'dejarse impregnar por los aires de los tiempos $^{4}$. Consecuentemente, este estudio se centra en la historia reciente de la introducción de las NTIC desde los documentos oficiales donde se examina el discurso dentro del campo del cambio tecnológico en general y en educación en particular. El proceso describe e interpreta los significados de documentos oficiales dentro de un contexto históricamente determinado que implica la configuración de representaciones de las NTIC a nivel institucional y social a través del discurso. El carácter de diseño emergente implica un enfoque interpretativo donde se involucra más que un método un enfoque teórico-metodológico. De esta manera, mediante el análisis de los documentos como una técnica de investigación histórica se intenta realizar una aproximación al objeto de investigación: los inicios de las NTIC desde los documentos oficiales gubernamentales para la educación. Este trabajo de carácter interpretativo se realiza bajo el supuesto de que no hay un análisis interpretativo único, sino que el proceso está abierto a la dinámica de la comprensión-explicación donde los análisis son susceptibles de cambio, encauzamiento y reinterpretación, aunque moderados especialmente por el contexto.

Julio Aróstegui, La investigación histórica: teoría y método (Barcelona: Crítica, 2001), 380.

Jean-Pierre Deslauriers, Investigación cualitativa: guía práctica (Pereira: Editorial Papiro, 2004), 5. 

información y la comunicación en la educación colombiana

\section{La globalización como contexto del cambio tecnológico}

La globalización no es necesariamente un concepto nuevo, pero sí su uso contemporáneo. Este término ha sido clave como justificación en el diseño de las nuevas políticas educativas de reforma tanto en los países industrializados como en los del llamado 'Tercer Mundo' debido a que ha adquirido una posición central en casi todas las manifestaciones del quehacer humano en el mundo actual. Cualquiera que sea la posición que se tenga sobre este concepto, difícilmente puede ignorarse. De ahí que su sentido puede tener diferentes implicaciones políticas, sociales, económicas, tecnológicas y por ende, educativas.

En efecto, el término como tal puede tener varios sentidos y no necesariamente corresponde, como pareciera, a un término nuevo, fruto de una moda académica o política sino al carácter plural e inaprehensible de un significante tan importante como para tenerse por contexto histórico actual. Sin embargo, es en la variedad del espectro de significados que se abre a las posibilidades de su uso ${ }^{5}$ y que tiende a ser aprovechado desde diferentes campos y situaciones (político, económico, social, educativo, tecnológico, etc.)

Es generalmente discutible si la globalización es una nueva época histórica o solo el remozamiento forzado de una estructura capitalista con características neoliberales existente en el mundo actual ${ }^{6}$. Sin lugar a dudas la globalización como fenómeno de la segunda mitad del siglo XX tiene que ver con rasgos de períodos históricos como la modernidad, el imperialismo, el capitalismo tardío, el neoliberalismo, y su vínculo con la internacionalización, la homogeneización y la multiculturalidad, esta última como el encuentro de los diferentes pueblos y culturas gracias al avance de las ciencias y al desarrollo tecnológico en el mundo.

Sin embargo, una característica que sobresale sobremanera es la relación entre la globalización y los aspectos sociales y culturales como preocupa-

Michael Halliday, El lenguaje como semiótica social (México: Fondo de Cultura Económica, 1982) 17; Ludwig Wittgenstein, Investigaciones filosóficas (Barcelona: Crítica, 1988), 184.

6 Eve Chiapello y Norman Fairclough, "Understanding the new management ideology: a transdisciplinary contribution from critical discourse analysis and new sociology of capitalism", Discourse and Society. Vol: 13 No. 2 (2002), 185-208. 
ción por la adecuación para el cambio y la "flexibilidad" al nuevo contexto que implica una tendencia a la internacionalización y la homogeneización de diferente tipo (político, social, cultural, etc.). Sin embargo, y quizás en forma más importante, algunos investigadores incorporan a la globalización características más de tipo económico y tecnológico ${ }^{7}$, estableciendo así un nuevo paradigma tecno-económico del conocimiento ${ }^{8}$. Esto ha dado como resultado el surgimiento de la "sociedad de la información" y la 'sociedad del conocimiento' como 'sociedad en red" con el ingrediente paradójico de que la globalización al mismo tiempo que 'conecta' gentes y territorios 'desconecta' implacablemente a personas, instituciones, países y sociedades que no se ajusten a lo que la razón hegemónica del mercado y la tecnología, (neoliberalismo, homogeneización) exige e impone y que a final de cuentas se considera como la base del desarrollo ${ }^{10}$.

En un sentido más general se destaca la globalización como el predominio de la conceptualización occidentalista que conlleva paradójicamente de la desintegración nacional, regional ("lo local") para dar vía a la integración transnacional ('lo global') ${ }^{11}$. Para unos analistas que observan la sociedad global y neoliberal desde una perspectiva crítica consideran la globalización como un punto de partida hacia la construcción de una 'sociedad de consumo' motivada por una forma de economía de mercado con mucho más poder que inclusive el mismo Estado ${ }^{12}$. Para otros, esta aparece como una catástrofe que amenaza las civilizaciones y culturas de las minorías ${ }^{13}$; y aun para otros, como parte de un férreo condicionamien-

\footnotetext{
Fermand Braudel, Escritos sobre la historia (Madrid: Alianza, 1991), 25; Marshall McLuhan, La galaxia Gutemberg: génesis del Homo typographicus (Barcelona: Planeta-Agostini, 1985). Ver: Marshall McLuhan, Comprender los medios de comunicación: las extensiones del ser humano (Barcelona: Paidós, 1996) y Richard Smith, Brian Lewis y Christine Massey, "Policy processes for technological change", en Knowledge management and higher education: a critical analysis, ed. Amy Metcalfe (Hershey: Information Science Pub., 2006), 182-195).

8 Michael Gibbons, et al., La nueva producción del conocimiento: la dinámica de la ciencia y la investigación en las sociedades contemporáneas (Barcelona: Pomares-Corredor, 1997), 119.

9 Ver: Manuel Castells, La era de la información: economía, sociedad y cultura. Vol. 1; La sociedad red. Vol. 2; El poder de la identidad; y Fin de milenio. Vol. 3 (Madrid: Alianza Editorial, 1998, 1999).

10 Noam Chomsky y Antonio Desmonts, El beneficio es lo que cuenta: neoliberalismo y orden global. (Barcelona: Crítica, 2000); Jesús Martín-Barbero, "Una mirada latinoamericana a la sociedad de la información”, en Desafios de la sociedad de la información en América Latina y Europa. Ed. UNICOM (Santiago de Chile: Editorial LOM, 2000), 27-33.

11 David Harvey, La condición de la posmodernidad (Buenos Aires: Amorrortu, 1990), 8.

12 Noam Chomsky y Heinz Dieterich, La sociedad global: educación, mercado y democracia (Tlalnepantla, México: Contrapuntos, 2002), 78.

13 Michael Apple, "Freire, Neo-liberalism and education", Discourse: Studies in the Cultural Politics of Education. Vol. 20 No. 1 (1999), 5; ver: Stanley Aronowitz, et. al., Tecnociencia y cibercultura: la interrelación entre cultura, tecnología y ciencia. (Barcelona: Paidós, 1998).
} 
to del poder político y económico de países altamente desarrollados hacia países más débiles en su entrada al sistema político y económico mundial, con sus dificultades y riesgos, especialmente en lo que tiene que ver con la educación ${ }^{14}$.

\section{Las nuevas tecnologías de la información y la comunicación (NTIC)}

Las tecnologías de la información y la comunicación han existido con el desarrollo de la humanidad misma y se refieren en forma más específica a las herramientas o medios para la comunicación entre los seres humanos. Las tecnologías modernas de la información y la comunicación han llegado en forma más reciente con la invención de la imprenta en el siglo XV, con la revolución industrial de finales del siglo XVIII y con la posterior invención del telégrafo, el teléfono y el desarrollo de la tecnología electrónica de nuestra época: la radio, la televisión, y la telefonía presentándose una orientación tecnológica de la globalización, es decir una globalización tecnológica.

En forma más reciente, se habla de las nuevas tecnologías de la información y la comunicación (NTIC). Estas se identifican como los sistemas normalmente conocidos dentro del campo de la 'informática' en la forma de la aplicación y uso de computadores, minicomputadores y computadores personales al procesamiento electrónico y digital de datos. También hacen parte de estas el desarrollo de la telefonía y su integración en redes mundiales de información y en procesos de comunicación en red, como en el caso de la Internet. Es en esta etapa en la que debido al veloz desarrollo tecnológico los cambios se han hecho más abruptos, y donde el fenómeno de la globalización ha tenido en la tecnología su catalizador más importante, es decir, la posibilidad de eliminar las fronteras físicas de tiempo y espacio, abarcando más espacio y 'conectando’ más territorios y personas a través de medios de comunicación más poderosos, 'asequibles' y 'flexibles'.

14 Paulo Freire, Cartas a Cristina: reflexiones sobre mi vida y mi trabajo (México: Siglo XXI, 1996), 14; Néstor García Canclini, La globalización imaginada (Buenos Aires: Paidós, 1999), 23. 


\section{Las NTIC en Colombia}

Las NTIC han llegado a Colombia principalmente desde el exterior y se han introducido en casi todas las manifestaciones de la vida diaria y la cultura. En educación, las entidades gubernamentales se han encargado de formalizar esta introducción a través de iniciativas oficiales, haciendo propio, en cierto grado, el desarrollo tecnológico que llega de otros países. Sin embargo, dado el alto grado de aceptación e integración que tienen las NTIC en la sociedad este proceso no está exento de influencias de diversa índole (económica y política) dentro del amplio marco de lo que hoy se denomina la 'sociedad de la información' y la 'sociedad del conocimiento' en la era de la globalización.

Por otro lado, estas innovaciones tecnológicas no han tenido la reflexión y el debate suficiente, no solo en cuanto a sus capacidades y sus limitaciones en educación sino, sobre todo en su incidencia en la naturaleza de la educación misma, sobre sus metas, propósitos y funciones en la 'era digital' en el ámbito de la internacionalización de finales del siglo XX. Todo esto ha generado un nuevo discurso de cambio e innovación tecnológica tanto en instituciones de educación básica y media como de educación superior lo cual facilita la introducción, familiarización y eventualmente integración de estas nuevas tecnologías en el sistema educativo del país. Dado que este proceso es muy reciente en Colombia y prácticamente no se ha realizado una aproximación al mismo desde una perspectiva histórica, analítica y reflexiva (mucho menos, crítica) es necesario preguntarse desde los documentos oficiales producidos por las instituciones gubernamentales sobre los inicios de este fenómeno en Colombia.

\section{Antecedentes de la introducción de las NTIC}

Los estudios que tienen como referencia los inicios documentales de las NTIC son bastante escasos en nuestro país. Sin embargo, se pueden detectar apreciaciones cercanas a las relaciones de estas con otros campos de influencia. Víctor Gómez sostiene, aunque en forma acrítica, que son los grandes cambios de tipo social, económico, político y cultural los que están estrechamente relacionados con los desarrollos de la ciencia o son el resultado de los mismos. Al mismo tiempo introduce en el sector edu- 
cativo (desde la perspectiva tecnocéntrica) la noción de 'mercado' cuando se refiere a que la educación es el 'procesamiento de información' de tipo intensivo. Consecuentemente, ve muy altas posibilidades de la informática como herramienta educativa innovadora para introducirse en ese 'mercado educativo' 15 .

Desde una perspectiva más socio-crítica, Antanas Mockus es uno de los pocos investigadores que aporta una mirada, de incipiente tenor reflexivo a la introducción y aplicación de las NTIC en educación por su carácter instrumentalista ${ }^{16}$. Utilizando una conceptualización integrada entre la teoría pragmática de la comunicación de Habermas ${ }^{17}$ y la socio-semiótica de Bernstein ${ }^{18}$, Mockus propende por una 'racionalización' de la educación donde se enfatice más la interacción comunicativa entre sujetos que el uso del medio tecnológico (NTIC) y sus connotaciones simbólicas. Asimila también la tecnología y su mal uso a un tipo de instrumentalismo no conveniente y prioriza la comunicación directa sobre el encasillamiento de la educación a un proceso técnico-instrumental, como el que se realiza en la producción económica o material (producción de bienes y servicios). En este sentido, se acerca a la crítica de las NTTC en el sentido de advertir sobre la irrupción de las mismas en forma abrupta dado que transformarían la enseñanza en proceso centrado en acumulación de datos y de proliferación de instrumentos tecnológico-educativos, configurándose de esta manera algo similar a 'medios de producción del conocimiento' a la manera del 'trabajo' y el 'mercado' con las consecuentes nociones de 'desempeño' y 'eficiencia' traídas de la concepción de producción desde la economía de mercado.

Dentro de la elaboración discursiva que se encuentra en el campo de los resultados de investigaciones sobre el impacto de las NTIC en educación, generalmente sobresalen apreciaciones eminentemente subjetivas. Por ejemplo, abundan los calificativos de 'mejor', más 'veloz', 'calidad' y 'signi-

15 Víctor Gómez, "Educación informática y educación informatizada", en Educadores e informática: promesas, dilemas y realidades (Santa Fe de Bogotá: Colciencias, 1988), 313-348.

16 Antanas Mockus, "Pedagogía, escritura e informática", en Educadores e informática: promesas, dilemas y realidades, ed. Víctor Gómez (Santa Fe de Bogotá: Colciencias, 1988) 103-153.

17 Jürgen Habermas, Teoría de la acción comunicativa: racionalidad de la acción y racionalización social, tomo 1, (Madrid: Taurus, 1999), 57.

18 Basil Bernstein, "Un ensayo sobre educación, control simbólico y prácticas sociales", en La construcción social del discurso pedagógico, ed. Mario Díaz (Bogotá: El Griot, 1993), 37-79; ver: Basil Bernstein, La estructura del discurso pedagógico: clases, códigos y control (Madrid: Morata, 1994), 38. 
ficativo', determinando así una valoración apriorística y tal vez excesiva de la innovación tecnológica en el campo educativo ${ }^{19}$. Desde una posición más reflexiva, estos mismos autores aseguran que frente a las NTIC el sujeto se circunscribe a la aplicación de procedimientos predefinidos, mas no a la evaluación y generación de conocimientos mediante la reflexión crítica sobre el fenómeno del aporte tecnológico en el cambio de la educación. Una de las observaciones más importantes está en el hecho de considerar las NTIC como formas de representación del mundo que mediante el nuevo discurso tecnocéntrico sirven para acercarse a una nueva realidad educativa, una realidad ligada al mundo de la vida 'global' como forma de pensamiento dominante.

Como se puede apreciar, la posición crítica sobre el cambio tecnológico de las NTIC en la educación es relativamente escasa en el nivel local debido al carácter introductorio y novedoso (alta validez física) de las nuevas tecnologías y los altos niveles de aceptación que han generado. Por un lado, la innovación tecnológica conlleva, en el conjunto de la sociedad y sus representaciones, al ideal de modernización, progreso, bienestar, y solución 'precisa' y 'eficaz' de los problemas individuales y sociales; por el otro, a la incuestionable penetración de la informática y las telecomunicaciones en el ámbito general, profesional y de la vida diaria como un indicio de la alta aceptación que tiene la tecnología en la mentalidad e imaginario de la gente, y especialmente de la gente joven.

\section{Los documentos oficiales sobre la introducción e implementa- ción de las NTIC en educación en Colombia}

Para afrontar documentalmente la pregunta de investigación después de hacer mención inicial de sus características de contexto arriba expuestos dentro del ámbito global y con el ánimo de la aproximación a la documentación oficial para el trabajo investigativo, se consideró pertinente utilizar los archivos gubernamentales donde reposan los documentos oficiales sobre las políticas de introducción e implementación tecnológica para el Estado, en este caso, el Archivo de la Presidencia de la República,

19 Luis Maldonado y Paola Maldonado, Nuevas tecnologías aplicadas a la educación: estado del arte de la investigación, 1990-1999 (vol. I) (Santa Fe de Bogotá: ICFES, COLCIENCIAS, Sociedad Colombiana de Pedagogía - SOCOLPE, 2001), 8. 
en una primera etapa y el Archivo General de la Nación en la segunda fase de este trabajo.

Una de las razones para utilizar el archivo de la Presidencia de la República es que en él reposan la correspondencia de los funcionarios del Estado, las actas, discusiones, propuestas, convenios, y en general las políticas de los gobernantes de turno, entre otros, con las que el gobierno colombiano de la época promovió la modernización del Estado en sus diferentes aspectos, entre ellos el educativo. Se puede determinar en primer término el proceso administrativo que llevó al diseño y estructuración de una política de las NTIC para modernizar el aparato administrativo y productivo del Estado, y en particular el sistema educativo. Todo esto en consonancia con los acontecimientos y desarrollos de vanguardia en otras partes del mundo en este campo, como en los Estados Unidos, Japón, Francia, y el Reino Unido.

\section{El origen del proyecto de los Centros de Informática}

Es particularmente interesante notar cómo desde comienzos de la década de los ochenta ya el gobierno colombiano había hecho contactos y provisiones para adelantar todo un proceso de capacitación del recurso humano e implementación de las NTIC para el desarrollo tecnológico, científico, educativo y social del país.

El gobierno del doctor Belisario Betancur (1982-1986) acudió a otros estados, gobiernos, centros e instituciones de trayectoria internacional en los campos tanto tecnológico como educativo, especialmente en lo que atañe a los distintos aspectos de la informática como el Instituto Tecnológico de Massachusetts MIT, de Estados Unidos y el Centro Mundial de Informática y Recursos Humanos, de Francia. Estos respondieron positivamente al proyecto modernizador del gobierno colombiano ${ }^{20}$.

Es así como una de las primeras iniciativas del gobierno fue la solicitud de apoyo logístico para la consecución de recursos e infraestructura a

20 "Oficio del Dr. Alonso Ospina Ospina al Sr. Embajador del Japón” (3 de junio de 1983) Archivo de la Presidencia de la República de Colombia (APRC), vol. 1, leg. 24 (Embajada Japón), f. 1. (En este archivo se puede encontrar también la nomenclatura de 'Caja', 'Carpeta' y folio en lugar de vol. leg. y f.) 
través de canales diplomáticos con el gobierno de los Estados Unidos y del Japón. Esto dio como resultado una primera donación del gobierno japonés en el área de la infraestructura informática para lo cual se incluyeron varias metas dentro de un ambicioso programa de implementación y de capacitación no solo en el sector de la administración pública sino también, y más importante, en el campo educativo.

En 1983, en carta del Sr. Secretario de la Presidencia de la República de Colombia, Dr. Alonso Ospina Ospina se exponía ante el Sr. Embajador de Japón los objetivos del mencionado programa para hacer uso de una importante donación de este país, en donde prima discursivamente el ámbito social y educativo:

Familiarizar a todos los colombianos con el computador personal de manera que este se transforme en una herramienta de trabajo, no sólo para ser usado en las oficinas, sino en los pequeños negocios y aun en los hogares.

Crear incentivos de manera que las instituciones de educación pública y privada adquieran mini y microcomputadores permitiendo el uso de ellos a todos los estudiantes.

Introducir dentro de los programas educativos del gobierno la tecnología informática y la telemática.

Impulsar programas especiales para los niños y jóvenes con dificultades en el aprendizaje.

Introducir el computador en el sector rural de manera que ayude a los granjeros a planear, controlar y administrar sus cultivos ${ }^{21}$.

El programa ambicionaba la modernización del país, en sectores tan importantes como la administración del Estado, la producción económica, el desarrollo rural, la educación y el bienestar de todos los colombianos. Cabe anotar que el diseño de la propuesta incluía no solo la implementación de la infraestructura en general sino más importante, la capacitación del personal que estaría a cargo del manejo de los equipos y que a su vez estaría idealmente multiplicando su experiencia y conocimientos con los demás de su campo y con la comunidad en general.

Es importante también recalcar el valor que el país le dio al componente social como parte central dentro del desarrollo tecnológico, es decir,

${ }_{21}$ “Oficio del Dr. Alonso Ospina Ospina al Sr. Embajador del Japón” f. 1, 2. 
el compromiso educativo y social a cuyo servicio debía ponerse la tecnología; tal era el reto de la informática (NTIC ahora) para con los sistemas educativo y social. En otras palabras, el discurso del gobierno era en este sentido condescendiente tanto en lo económico como en lo social. Así lo expresaba en su carta el doctor Alfonso Ospina Ospina, secretario general de la presidencia en su propuesta al Dr. Nagasaki, embajador del Japón ${ }^{22}$,

Dentro de este contexto, el uso de la informática es uno de los desarrollos actuales más importantes ya que su aplicación en el campo de la educación, la salud, la agricultura y de la industria, contribuirán significativamente al progreso social y económico de nuestra sociedad ${ }^{3}$.

Todo esto se enmarcaba evidentemente dentro de un esquema programático como fue el "Plan Nacional de Desarrollo" (1983-1986) base de los procesos educativo y productivo del país, dos conceptos tan dispares hoy que en un momento dado se unieron en un discurso coherente y comprometido con las necesidades de desarrollo integral del país y de la sociedad. De esta manera, nacía el proyecto de "Centros de Informática"24, producto de una donación del gobierno del Japón consistente en 100 equipos de computación modernos “...los cuales se instalarían en las capitales de departamentos y de algunos municipios" ${ }^{\prime 25}$.

El gobierno acudió entonces a las instancias internacionales que imponían el liderazgo en los aspectos de la tecnología informática relacionada con la economía, la industria y la educación en el mundo.

Dentro de este esquema programático del gobierno sobre las NTIC también se incluía como participante o receptor de estas tecnologías para su capacitación, a la niñez del país:

"Los centros de microcomputadores que se van a instalar en las ciudades del país y en algunos municipios tienen por objeto familiarizar a los colombianos y en especial a los NIÑOS con los computadores ${ }^{\prime 26}$. (Énfasis mí)

"Oficio del Dr. Alonso Ospina Ospina al Sr. Embajador del Japón" f. 1, 2.

"Oficio del Dr. Alonso Ospina Ospina al Sr. Embajador del Japón" f. 1.

"Oficio del Dr. Alonso Ospina Ospina al Sr. Embajador del Japón" f. 3. Oficio del Dr. Hernán Heltz Peralta al Sr.

Embajador del Japón Hiroshi Nagasaki. (15 de julio de 1983). (APRC). Vol. 1, leg. 24 (Embajada Japón), f. 3.

APRC, vol. 1, leg. 24 (Embajada Japón), f. 6.

APRC, vol. 1, leg. 24 (Embajada Japón), f. 7. 
Se puede decir que el gobierno también se asesoró muy bien de expertos reconocidos internacionalmente en el campo educativo sobre la transferencia tecnológica y de la capacitación del recurso humano. Personajes de reconocida trayectoria en los campos del software, de hardware y de su aplicación en educación provenientes de importantes instituciones mundiales se hicieron presentes en la convocatoria del gobierno, como por ejemplo, los doctores Kinya Matzumura, y Hiroyuki Saito del Japón. El gobierno también hizo contactos, para los planes de capacitación, con el Instituto Tecnológico de Massachusetts (MIT) de los Estados Unidos, y particularmente con autoridades como el Doctor Seymour Papert, el Doctor Nicholas Negroponte, y otros expertos norteamericanos en el campo de las tecnologías de la información y su aplicación en educación y en la ciencia cognitiva que ya empezaba a destacarse a nivel internacional con la ayuda del nuevo ingrediente tecnológico. De la misma manera, pero en menor proporción, se invitó por parte de estos gobiernos al personal colombiano para realizar entrenamiento y capacitación en el exterior.

En este orden de ideas, es muy ilustrativo el convenio de investigación realizado entre el Instituto Tecnológico de Massachusetts y el gobierno colombiano establecido inicialmente como proyecto formal ${ }^{27}$ en 1982 en el cual resalta la parte educativa e investigativa:

'Las partes aqui mencionadas se comprometen a lo siguiente:

1. DECLARACIÓN DE TRABAJO. MIT se compromete a hacer su mejor esfuerzo para desarrollar el programa de investigación denominado "Computación personal para el desarrollo colombiano" como se describe en la propuesta adjunta.

2. INVESTIGADOR PRINCIPAL. La investigación será supervisada por los profesores Nicholas Negroponte y Seymour Papert. Si, por cualquier razón, no pudieran continuar sirviendo como investigadores principales y un sucesor aceptable para ambas partes, MIT y el patrocinador, no está disponible, este convenio se dará por terminado como se prevé en el artículo 6"28. (Mi traducción)

Se puede notar que la introducción e implementación de las NTIC en educación por parte del gobierno colombiano no fue un hecho fortuito o

27 "Acuerdo de investigación de MIT por Dr. George H. Drummer" (enero 3, 1983) (APRC), vol. 1, leg. 3 (M.I.T.), f. 12. Este es el documento del acuerdo de investigación presentado por el Massachusetts Institute of Technology representado por el Dr. George H. Drummer, Director de programas de apoyo, redactado en julio de 1982.

28 "Acuerdo de investigación de MIT por Dr. George H. Drummer" f. 12. 
un proceso signado inicialmente por la presión mercantilista globalizante inicial o por la presión de estar a la par con otros estados y sistemas desarrollados, sino por el contrario, tenía en su centro toda una concepción de desarrollo integral, donde se contemplaba la situación económica, industrial, educativa y social del país. Pero particularmente, como se ha visto en el documento anterior, todo este proceso que fue el fruto de esfuerzos institucionales de política gubernamental y de un discurso modernizante manejado por sus representantes estaba enmarcado teóricamente dentro de aspectos de investigación, de cooperación y más importante dentro de la capacitación del recurso humano colombiano.

\section{La política informática del país}

A comienzos de la década de los ochenta el gobierno colombiano consciente de la importancia de estas nuevas tecnologías para el desarrollo tecnológico, económico y social del país y de la importancia de la información y su manejo en la planificación y la gestión de la administración, la producción y la investigación promovió la creación de varias entidades estatales, a saber, el "Consejo Nacional de informática", la "Secretaría de informática de la Presidencia de la República", el "Plan Nacional de Informática" y el establecimiento del "Centro Latinoamericano de Recursos Humanos e Informática"29. No se ve explícitamente ningún tipo de interés de tipo económico o político de parte de los actores extranjeros ya que en esta ocasión fue el gobierno colombiano quien acudió a las instituciones educativas internacionales principalmente. Sin embargo, es preciso manifestar que la puerta para las futuras intervenciones directas o indirectas de tipo externo había quedado abierta.

La política del gobierno en torno a la tecnología informática de la década de los ochenta (como se denominaban inicialmente en ese momento) estaba no solamente dirigida al sector productivo e industrial, sino también a la sociedad y a la educación en su conjunto para promover el desarrollo colectivo. También era consciente que se había perdido mucho tiempo y espacio en este campo de rápido desarrollo tecnológico, y consecuente-

29 "Oficio Desarrollo de Recursos Humanos y la Informática" (APRC), vol: 1, leg. 30 (Proyecto Ley de Informática), f. 82. Este es el documento oficial sobre Desarrollo de los Recursos Humanos y la Informática de la Presidencia de la República que se había promulgado mediante el decreto 146 del 24 de enero de 1983 y que inicialmente se había introducido como proyecto de Ley (Ley de informática) en marzo de 1984. 
mente, se tenía que actuar de manera rápida. Como se mencionó anteriormente, uno de los sectores que se beneficiarían de esta política fue necesariamente el de la educación y la salud: "Particular atención se otorgará a la modernización del Estado así como a la búsqueda del mejoramiento en programas de educación, agricultura, desarrollo rural y salud"30.

Algunas de las estrategias del plan de acción para la introducción y el conocimiento masivo de esta política de nuevas tecnologías comprendían, el establecimiento de 'salas de computadores' para uso libre y gratuito, la realización de seminarios de capacitación sobre temas actuales de informática, y el "establecimiento de incentivos para que las instituciones educativas pudieran adquirir equipos disponibles para todos los estudiantes"31.

Las áreas de cobertura y campos de incidencia de la política de informática del gobierno incluían tanto la educación formal como la no-formal y la alfabetización. Esto es, no solo se restringía a nivel de la educación básica y superior de tipo presencial sino también a la educación a distancia, a la instrucción vocacional, a la alfabetización y a los programas de rehabilitación. El campo de acción de la alfabetización y la capacitación e instrucción formales, incluía pues, si no toda, una muy buena parte del ámbito educativo.

Se ha podido establecer documentalmente que entre los planes del gobierno de entonces estaba incluida la educación asumiéndola como receptora de la tecnología para fines de capacitación, inicialmente, sin considerar todavía aspectos de enseñanza-aprendizaje. Inclusive se puede notar la preocupación por este componente en los programas de educación superior y de su incidencia sobre la preparación de los futuros profesionales para el desarrollo del país. Así lo daba a entender el texto del proyecto de ley que proponía la creación del "Consejo Nacional de Informática" cuando originalmente y específicamente mencionaba que "la Secretaría de Informática de la Presidencia de la República conceptuaría sobre los programas curriculares de estudios superiores y técnicos en informática desarrollados, coordinados u autorizados por los organismos estatales competentes" ${ }^{\prime 2}$.

\footnotetext{
30 "Oficio Desarrollo de Recursos Humanos y la Informática" (APRC), vol: 1, leg. 3 (M.I.T.) f. 12.

31 "Oficio Desarrollo de Recursos Humanos y la Informática" (APRC), vol: 1, leg. 3 (M.I.T.) f. 81.

32 "Oficio Borrador Proyecto de ley para la creación del Consejo Nacional de Informática" (Marzo, 1984). (APRC), vol. 1, leg. 30 (Proyecto Ley de Informática), f. 82.
} 
Era evidente entonces el interés del Estado por la capacitación del recurso humano en la preparación del contexto para el desarrollo industrial, social y educativo del país, no solamente en el conocimiento de estas tecnologías sino en la aplicación de las mismas en los diferentes campos del saber y el quehacer cotidianos.

\section{Las nuevas tecnologías de la información y la comunicación en la nueva Constitución de 1991}

El proceso histórico más reciente de las NTIC en Colombia tiene que ver con el discurso de los documentos que se produjeron en el gobierno del Doctor César Gaviria Trujillo como Presidente de la República a raíz de las discusiones, plenarias y deliberaciones de la Asamblea Nacional Constituyente de 1991, sobre el tema de la calidad de la educación y sobre los inicios del programa de modernización del Estado colombiano a través de las NTIC en el campo de la educación pública. Estos antecedentes servirían para entender cómo se desarrolló la preocupación del Estado por la modernización del sistema educativo, previa a las discusiones de trabajo de la Asamblea Nacional Constituyente y cuya documentación reposa en el Archivo General de la Nación ${ }^{33}$. Esto tiene una especial importancia dado que fueron los inicios conceptuales y discursivos manejados en este contexto y sobre los que se introdujeron las primeras nociones del desarrollo educativo global, el valor de la información y del conocimiento mayormente desde el ámbito internacional desde donde ya se habían dado pasos agigantados en el campo del cambio educativo mediado tecnológicamente.

Por otro lado, la Constitución de 1991 abría posibilidades insospechadas en este campo cuando destacaba, por ejemplo, el concepto discursivo de la internacionalización de la Educación Superior en Colombia. De esta manera la reforma a la legislación educativa de Colombia se había nutrido por el desarrollo de los derechos, metas, principios y valores que a nivel global se habían incorporado en la Carta Magna como ya era el caso en otros países. Bajo la nueva Constitución, el gobierno era responsable de este proceso ya que según el texto, el mismo Estado lo acercaría eventualmente a la educación: "El Estado promoverá la internacionalización de las

“Asamblea Nacional Constituyente” Archivo General de la Nación (1991), (AGN), Sección República, Constituciones, Asamblea Nacional Constituyente. 
relaciones políticas, económicas, sociales y ecológicas basadas en la equidad, reciprocidad e interés nacional" ${ }^{34}$. Por otra parte se puede establecer que los valores, principios y derechos fundamentales establecidos en la Constitución habían sido la base para asegurar profundos cambios en educación. En cuanto a la Ley 30 de 1992, se puede establecer bajo el amparo del concepto de 'globalización' la introducción del concepto de la 'internacionalización' como uno de los retos principales del sector educativo. Bajo esta ley, el Estado cedía responsabilidad y otorgaba autonomía a las institu-ciones de educación superior para estimular la capacitación, fortalecer las comunidades académicas y coordinar con otros órganos nacionales e internacionales los procesos de internacionalización y globalización de la educación. Consecuentemente, el objetivo central de esta ley con respecto a los vínculos con el mundo global era que la comunidad académica trascendiera las fronteras nacionales y de la misma manera, se infiere, se abriera a las demandas y presiones internacionales.

\section{El derecho a la educación y a la información}

De acuerdo con las plenarias y discusión en la Asamblea Nacional Constituyente en la comisión de derechos humanos, se destaca el trabajo emprendido por la subcomisión 014 sobre el 'Derecho a la Educación', fomento a la cultura, la ciencia y la tecnología. Su punto de partida se sustentaba en: "El derecho que tiene toda persona a crear, disfrutar y acceder a los bienes y valores de la cultura, entendida en la forma amplía que se acaba de explicar" ${ }^{\text {" }}$.

Vale la pena resaltar que en esta comisión que trató sobre el derecho a la educación y a las posibilidades de desarrollo humano, se hubieran integrado factores económicos al desarrollo de la cultura a través de los medios de comunicación. De esta forma se tiene que el concepto de tecnología estaba íntimamente relacionado con la de información que se podía manejar, desde contextos políticos, económicos, y sociales como el de 'sociedad del conocimiento' (como un aspecto de valor) y el de “internacionalización" de la economía, de la educación y de la cultura que se estaba manejando internacionalmente. El nuevo concepto de sociedad

\footnotetext{
34 Tomado del artículo 226 de la Constitución Política de Colombia. Capítulo VIII: De las relaciones internacionales; Título VII: De la Rama Ejecutiva.

35 "Tecnología: Artículo aditivo" (1991) (AGN), Sección República, Constituciones Asamblea Nacional Constituyente, vol. 04498, leg. 251, f. 116.
} 
como la "sociedad de la información", la "sociedad del conocimiento" y por consiguiente la "sociedad de la economía de consumo" y por ende, el de mercado no solo era el que utilizaba los bienes básicos y esenciales de sostenimiento sino aquel que además utilizaba los de la información y la comunicación en aspectos productivos. A este respecto manifestaba la comisión en cuestión:

\begin{abstract}
Nuestra época esta signada por una internacionalización económica y cultural que conduce hacia una comunidad de naciones basada en el conocimiento y la creación cultural. En particular, tanto el progreso tecnológico y el desarrollo de los medios masivos de comunicación, como preservación de la diversidad cultural requiere y genera procesos deliberados de creaciones, de apropiación y difusión de conocimientos y valores ${ }^{36}$.
\end{abstract}

Por consiguiente, se era consciente de las implicaciones de lo que se propugnaba. De ahí que era la voluntad de la comunidad representada en el trabajo de esta comisión compuesta por 32 miembros de un alto nivel de conocimiento y responsabilidad que haya relacionado el desarrollo económico y cultural con el avance del conocimiento a través del uso de las tecnologías de la información y la comunicación. En otras palabras, y como se manifestaba en el documento, el país "debe tener la capacidad de comprender y aprovechar las grandes tendencias contemporáneas" ${ }^{37}$. Es decir, se estaba implícitamente aludiendo al contexto de la globalización en el que las NTIC ya estaban jugando un papel importante en el desarrollo del mundo tecnológicamente orientado, y que lo haría notorio mayormente en el campo educativo con el paso del tiempo.

Referente a la acción y función de los medios masivos de comunicación es importante considerar el trabajo realizado por los constituyentes Abel Rodríguez y José Germán Toro al presentar el proyecto de la "reforma democrática de la educación", cuando en el artículo 11 proponían que los medios masivos de comunicación social tenían que estar obligados a "coadyuvar en la realización de los fines de la educación, en los términos que ordene la ley" "38. Se estaba propugnando, sin saberlo, la participación de lo que más tarde entraría a competir por una parte importante de la

\footnotetext{
36 “Tecnología: Artículo aditivo" (1991) (AGN), f. 118.

Tecnología: (1991) (AGN), f. 118.

38 "Reforma democrática de la Educación" (1991) Archivo General de la Nación, (AGN) Sección República, Constituciones, Asamblea Nacional Constituyente, vol. 02217, leg. 21, f. 7.
} 
educación, la Red Mundial (Internet) y los programas nacionales e internacionales de educación a distancia y virtuales.

Se suma a lo anterior, el derecho que la Constitución debía otorgar a todo ciudadano que deseara informarse voluntariamente y el derecho a ser informado en forma completa. Esto quería decir, que no solo era importante el 'querer' informarse sino que los instrumentos de acceso a la información debían estar disponibles para tal efecto. Con la disponibilidad de la infraestructura tecnológica a través de la cual se accede a la información ya sea análoga o digital, podría entonces darse viabilidad para que la persona pueda en realidad informarse. Esto se deduce del 'derecho a la comunicación' establecido en una propuesta sustitutiva que se había trabajado en el ámbito de la Asamblea Nacional Constituyente, sobre el tema:

"Toda persona tiene derecho a [...] informarse y ser informada de manera veraz, imparcial y completa" 39 .

Lo anterior implicaba también aspectos de comunicación social como derecho a la información y por ende al conocimiento y la responsabilidad de hacerlo en forma 'completa'. De esta manera, el discurso tecnocéntrico había sido preparado desde las fases de propuestas iniciales hasta las de tipo constitucional, las legales y oficiales para que pudiera desprenderse de él todo un proceso de introducción e implementación discursiva que en la práctica durante la década de los noventa solo se tendría eventualmente solo como un imaginario colectivo.

\section{Las universidades y las nuevas tecnologías de la información en educación}

La universidad colombiana no fue ajena al clamor de los intelectuales, de los grupos sociales y de la comunidad educativa en general para hacer saber sus inquietudes y exigencias en torno a la modernización de la educación y a la mejor implementación de una estructura educativa que garantizara el desarrollo integral de los ciudadanos para una época de un vertiginoso desarrollo tecnológico, informático y de telecomunicaciones.

39 "Universidades del Estado" Archivo General de la Nación (AGN), Sección República, Constituciones, Asamblea Nacional Constituyente, vol: 00997, leg. 45, f. 189. 
Es precisamente en ejercicio de la autonomía universitaria que se propuso la mejoría de la calidad de la educación superior a través de procesos de modernización, de internacionalización y del compromiso del gobierno en apoyar la investigación y el desarrollo científico y tecnológico de las regiones. La tecnología como un medio para que la universidad pudiera desempeñarse no solo como transmisora de conocimiento sino como generadora del mismo se podría lograr en forma significativa teniendo en las NTIC no solo una herramienta sino también un aliado y un medio o puente para que la comunidad académica y científica se proyectara hacia las demás naciones, pero al mismo tiempo se abriera a las mismas. En este sentido se pudo notar cómo organizaciones tales como la Asociación Colombiana de Universidades (ASCUN) presentó en estos términos su proyecto de reforma educativa ante la Asamblea Nacional Constituyente para su discusión ${ }^{40}$.

Con respecto a la importancia del papel de la información en la nueva sociedad del conocimiento, los organismos organizados, sindicatos e instituciones universitarias tomaron partido de las posibilidades de ser escuchados en el marco de la Asamblea Constituyente de 1991 y así enviaron sus proyectos y propuestas a las diferentes instancias, para ser formalmente incluidas en las agendas de discusión. Así mismo hubo participación en las plenarias en el contexto de la nueva constitución para exponer las consideraciones y posiciones de índole educativa con respecto a las tecnologías de la información y la comunicación y al papel que estas jugarían en el futuro del país. Las universidades públicas organizadas y representadas en ASCUN fueron una buena muestra de este proceso.

La apertura de la educación hacia el exterior a través del cambio tecnológico, o sea, en el sentido de tener posibilidades de afectar y ser afectada por los desarrollos políticos, económicos, sociales y tecnológicos de índole externa estaba dada (tal vez más en el segundo caso de las posibilidades que en el primero). En otras palabras el proceso de apertura con la internacionalización de la educación y en el contexto influyente de la globalización se había abierto paso, con todas sus posibles ventajas y también sus limitaciones y riesgos.

"Asociación Colombiana de Universidades ASCUN" (Marzo 1991) Archivo General de la Nación (AGN), vol. 02259, leg. 221. 


\section{CONCLUSIÓN}

No hay duda que el mundo presentó profundos cambios, políticos, económicos, sociales, tecnológicos y culturales a finales del siglo XX. Se ha discutido sobre el tipo de cambio en cada uno de estos ámbitos en la historia reciente de la sociedad moderna, particularmente en el contexto del fenómeno de la globalización y sus aspectos concomitantes como los que han guiado el mundo y que algunos analistas han denominado como perteneciente al desarrollo de una nueva fase del capitalismo, el nuevo capitalismo, o capitalismo tardío, entre otras denominaciones ${ }^{41}$.

Dentro de esta configuración del mundo político, económico, social y cultural resalta el aspecto tecnológico como de innegable influencia en la historia de la humanidad y la educación y que se ubica estrechamente con los anteriores aspectos en las nuevas denominaciones de la sociedad: 'sociedad de la información', 'sociedad del conocimiento', 'sociedad de la era digital', 'sociedad global', 'sociedad en red' en la que se manifiestan cambios de orden paradigmático como los pregonados desde la década de los sesenta por Marshall McLuhan, hasta la de los noventa por Manuel Castells. Sin embargo, estos cambios que se han producido dentro del fenómeno de la globalización desde las postrimerías del siglo pasado también han tocado el ámbito educativo, particularmente la Educación Superior y las universidades en casi todo el mundo y particularmente en el país, sujetas a fuertes presiones hacia el cambio como resultado de una compleja interacción de fuerzas internas pero especialmente externas estrechamente ligadas a la globalización tanto de tipo económico como tecnológico ${ }^{42}$ y el dominio del paradigma político-económico que se ha impulsado cada vez más desde las nuevas tecnologías de la información.

A pesar del relativo atraso del país en cuestiones de modernización con la introducción de las NTIC respecto a los demás países en vía de desarrollo, y del papel que estas deberían cumplir en términos de desa-

Jeremy FOX,Chomsky y la globalización (Barcelona: Editorial Gedisa, 2004), 5.

42 Richard Katz, "Competitive strategies for higher education in the information age",en Dancing with the devil: information technology and the new competition in higher education, ed. Richard Katz (San Francisco- Calif.: Jossey-Bass Publishers, 1999), 28-52. Ver : Richard Smith, Brian Lewis y Christine Massey "Policy processes for technological change", en Knowledge management and higher education: a critical analysis, Ed. Amy Metcalfe (Hershey: Information Science Pub., 2005), 182-195. 
rrollo, es importante considerar que desde comienzos de la década de los ochenta ya se estaban 'oficializando' discursivamente las gestiones de su inicio. Es importante destacar la visión, actitud y gestión positiva que se tuvieron por parte del Estado colombiano no solo en el sentido de agilizar los mecanismos oficiales, legales e institucionales para la introducción discursiva y documental de las NTIC en el aparato del Estado sino también en lo que concierne a la modernización de la economía y el desarrollo integral del país, con la creación de diferentes organismos y entidades necesarios para su gestión, en la práctica.

Son precisamente los niveles social y educativo los que se destacan en este breve estudio, en la forma que se esperaría a nivel de las políticas del gobierno para oficialmente llevar a cabo los primeros pasos que configuraron discursivamente un importante cambio en la preparación y formación de los futuros profesionales y de la sociedad que se integrarían a la fuerza productiva y laboral del país. Esto en lo que concierne a la parte oficial a nivel de los documentos gubernamentales en los que se asumen procesos de tanta responsabilidad como el de la modernización, la innovación, el desarrollo social, y en últimas el cambio mismo.

Sin embargo, a pesar de la diligente actitud y acción de las políticas de Estado en los gobiernos de turno desde inicios de este proceso a comienzos de la década de 1980 hasta su configuración más moderna en el marco de la Constitución de 1991, la introducción oficial de las NTIC con la apertura del país en cuanto a implementación y enriquecimiento educativo y cultural también se dio desde la otra vía, la afectación no solo tecnológica y educativa sino en la introducción de paradigmas políticos, económicos y culturales de tipo exógeno.

Por consiguiente, para una futura comprensión de este proceso sería importante conocer si todas estas iniciativas, entre otras, tuvieron la manifestación real en la práctica y determinar históricamente cuál ha sido su desarrollo posterior y sus implicaciones reales; cuáles han sido las que se consolidaron; y en qué medida han afectado las prácticas educativas y culturales de las gentes de nuestra época actual con respecto a su desarrollo educativo, económico y social. De la misma manera se necesitaría conocer cuáles han sido las implicaciones reales de esta forma de introducción 
discursiva a la modernización del Estado y consecuentemente de la educación en sus diferentes niveles paralelamente al desarrollo tanto económico, como social del país.

Finalmente, no se podría dejar de lado el aspecto crítico de este proceso, especialmente en el contexto histórico del cambio tecnológico que supone la articulación del fenómeno de la globalización con la economía de mercado y el desarrollo social que propende a la vez por la multiculturalidad y la homogeneidad (estandarización) como motivación tecno-económica de nuestra época. La mirada crítica sobre el cambio tecnológico en educación permitiría entrever si hay algo más allá de la convicción de 'sentido común’ sobre los beneficios históricos del cambio tecnológico en educación. Esto tendría que ver con los medios discursivos que apoyan la difusión del cambio tecnológico en educación, su introducción, acoplamiento e integración a las formas tradicionales de educación con el incremento de formas tal vez no muy coincidentes de acción y que llevaría eventualmente a que se tengan que cambiar las metas, misión y visión educativas que han preocupado tanto al ser humano y a las que se ha dedicado mucho más tiempo y esfuerzo que el que lleva el desarrollo tecnológico moderno.

\section{FUENTES}

Archivo de la Presidencia de la República de Colombia (APRC), Bogotá-Colombia. Caja 1, leg. 24 (Embajada de Japón), f. 1.

Archivo de la Presidencia de la República de Colombia (APRC), Bogotá-Colombia. Caja 1, leg. 24 (Embajada de Japón). f. 3.

Archivo de la Presidencia de la República de Colombia (APRC), Bogotá-Colombia. Caja 1, leg. 3 (M.I.T.), f. 12.

Archivo de la Presidencia de la República de Colombia (APRC), Bogotá-Colombia. Caja 1, leg. 30 (Proyecto Ley de Informática), f. 82.

Archivo de la Presidencia de la República de Colombia (APRC), Bogotá-Colombia. Borrador del Proyecto de ley para la creación del Consejo Nacional de Informática, Caja 1, leg. 30 (Proyecto Ley de Informática), folio 82.

Archivo General de la Nación (AGN), Bogotá-Colombia. Sección República, Constituciones, Asamblea Nacional Constituyente, 1991.

Archivo General de la Nación (AGN), Bogotá-Colombia. Sección República, Constituciones Asamblea Nacional Constituyente de 1991. 
Los comienzos oficiales del uso de las nuevas tecnologías de la información y la comunicación en la educación colombiana

Archivo General de la Nación (AGN), Bogotá-Colombia. Sección República, Constituciones, Asamblea Nacional Constituyente:

Asociación colombiana de Universidades ASCUN.

\section{REFERENCIAS}

Apple, Michael. "Freire, Neo-liberalism and education". Discourse: Studies in the Cultural Politics of Education, vol. 20 No. 1 (1999): 5.

Aronowitz, Stanley, Barbara Martinsons y Michael Menser. Tecnociencia y cibercultura: la interrelación entre cultura, tecnología y ciencia. Barcelona: Paidós, 1998.

Aróstegui, Julio. La investigación histórica: teoría y método. Barcelona: Crítica, 2001.

Bernstein, Basil. "Un ensayo sobre educación, control simbólico y prácticas sociales”, en La construcción social del discurso pedagógico, editado por Mario Díaz. Bogotá: El Griot, 1993, 37-79.

Bernstein, Basil. La estructura del discurso pedagógico: clases, códigos y control. Madrid: Morata, Fundación Paideia, 1994.

Braudel, Fernand. Escritos sobre la historia. Madrid: Alianza, 1991.

Castells, Manuel. La era de la información: economía, sociedad y cultura. La sociedad red, vol. 1, Madrid: Alianza, 1998.

Castells, Manuel. La era de la información: economia, sociedad y cultura. El poder de la identidad, vol. 2. Madrid: Alianza, 1998.

Castells, Manuel. La era de la información: economía, sociedad y cultura. Fin de milenio, vol. Madrid: Alianza, 1999.

Chiapello, Eve, y Norman Fairclough. "Understanding the new management ideology: a transdisciplinary contribution from critical discourse analysis and new sociology of capitalism". Discourse and Society, vol. 13 No. 2 (2002): 185-208.

Chomsky, Noam, y Antonio Desmonts. El beneficio es lo que cuenta: neoliberalismo y orden global. Barcelona: Crítica, 2000.

Chomsky, Noam, y Heinz Dieterich. La sociedad global: educación, mercado y democracia. México: Contrapuntos, 2002.

Deslauriers, Jean-Pierre. Investigación cualitativa: guía práctica. Pereira: Editorial Papiro, 2004.

Fox, Jeremy. Chomsky y la globalización. Barcelona: Editorial Gedisa, 2004.

Freire, Paulo. Cartas a Cristina: reflexiones sobre mi vida y mi trabajo. México: Siglo XXI, 1996.

García Canclini, Néstor. La globalización imaginada. Buenos Aires: Paidós, 1999.

Gibbons, Michael, Camille Limoges, Helga Nowotny, Simon Schwartzman, Peter Scott y Martin Trow. La nueva producción del conocimiento: la dinámica de la ciencia y la investigación en las sociedades contemporáneas. Barcelona: Pomares-Corredor, 1997. 
Gómez, Víctor. "Educación informática y educación informatizada", en Educadores e Informática: promesas, dilemas y realidades, editado por Víctor Gómez. Santa Fe de Bogotá: Colciencias, 1988, 313-348.

Habermas, Jürgen. Teoría de la acción comunicativa, racionalidad de la acción y racionalización social, vol. 1. Madrid: Taurus, 1999.

Halliday, Michael. El lenguaje como semiótica social. México: Fondo de Cultura Económica, 1982.

Harvey, David. La condición de la posmodernidad. Buenos Aires: Amorrortu, 1990.

Katz, Richard. "Competitive strategies for higher education in the information age", en Dancing with the devil: information technology and the new competition in higher education, editado por Richard Katz. San Francisco- Calif.: Jossey-Bass, 1999, 28-52.

Maldonado, Luis, y Paola Maldonado. Nuevas tecnologías aplicadas a la educación: estado del arte de la investigación, 1990-1999. Santa Fe de Bogotá: ICFES, COLCIENCIAS, Sociedad

Colombiana de Pedagogía, 2001. Martín Barbero, Jesús. "Una mirada latinoamericana a la sociedad de la información", en Desafíos de la sociedad de la información en América Latina y Europa, editado por UNICOM. Santiago de Chile: Editorial LOM, 2000, 27-33.

McLuhan, Marshall. La galaxia Gutemberg: génesis del Homo typographicus. Barcelona: PlanetaAgostini, 1985.

McLuhan, Marshall. Comprender los medios de comunicación: las extensiones del ser humano. Barcelona: Paidós, 1996.

Mockus, Antanas. "Pedagogía, escritura e informática", en Educadores e informática: promesas, dilemas y realidades, editado por Víctor Gómez. Santa Fe de Bogotá: Colciencias, 1988, 103-153.

Smith, Richard, Brian Lewis y Christine Massey. "Policy processes for technological change", en Knowledge management and higher education: a critical analysis, editado por Amy.

Metcalfe. Hershey: Information Science Pub. 2005.

Wittgenstein, Ludwig. Investigaciones filosóficas. Barcelona: Crítica, 1988.

\begin{tabular}{|c|}
\hline $\begin{array}{l}\text { Benavides B., Jorge E. "Los comienzos oficiales del uso de las nuevas } \\
\text { tecnologías de la información y la comunicación en la educación } \\
\text { colombiana". Revista Historia de la Educación Latinoamericana. Vol. } 14 \\
\text { No, 19, (2012): }\end{array}$ \\
\hline
\end{tabular}

